The measurement of X-ray intensity and the necessity for an international method

This content has been downloaded from IOPscience. Please scroll down to see the full text. 1922 Proc. Phys. Soc. London 35 5D

(http://iopscience.iop.org/1478-7814/35/1/343)

View the table of contents for this issue, or go to the journal homepage for more

Download details:

IP Address: 130.237.165.40

This content was downloaded on 27/08/2015 at $21: 20$

Please note that terms and conditions apply. 


\title{
THE MEASUREMENT OF X-RAY INTENSITY AND THE NECESSITY FOR AN INTERNATIONAL METHOD.
} BY

\author{
S. Russ, D.Sc., F.Inst.P., Physics Dept., Middlesex Hospital.
}

FHySICISTS rely mainly upon ionisation methods for the measurement of X-ray intensity, and a very large variety of devices is now available by which the intensity rof a beam of $\mathrm{X}$-rays can be gauged.

It is imfcrtant that the instrument should be equally reliable for the measurement of X-rays of different wave-lengths. This is not at all an easy matter because of the complicated phenomena which affear when a primary beam of X-rays enters an absorbing medium. Let us consider a beam of X-rays of intensity $I_{0}$ and wavelength $\lambda_{0}$ entering an air space of length $x$ (Fig. 1). The intensity is defined as the anount of energy incident fer sccond ufon unit area placed normal to the rays.

On entering this space scme of the radiation is scattered, some is absorbed, and the remainder is transmitted. We may write this as follows :-

$$
I_{0}=s I_{0}+s^{\prime} I_{0}+q_{0}+I_{0} e^{-\mu_{0} x} \text {, }
$$

where $s$ and $s^{\prime}$ are the scattering coefficients in the backward and forward directions, $q_{0}$ is the quantity of energy quenched per second in the air space and causing ionisa-

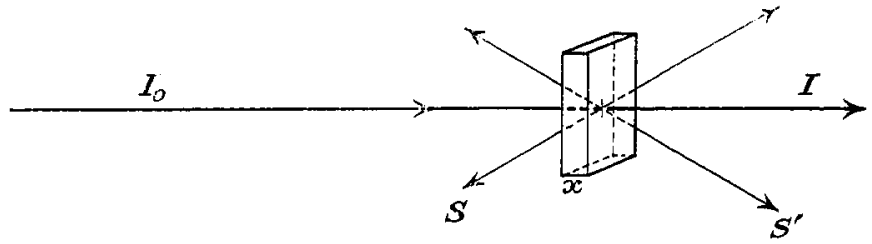

FIG. 1.-INTENSITY EFFECT METER.

tion, and the last term is the fraction transmitted, $\mu_{0}$ being the absorption coefficient of the medium for the wave-length specified; heating effects are neglected here.

Consider now a beam of different wave-length $\lambda_{1}$ and of intensity $I_{1}$, then, as before, we have -

$$
I_{1}=s_{1} I_{1}+s_{1}^{\prime} I_{1}+q_{1}+I_{1} e^{-\mu_{1} x} \text {. }
$$

Now it is possible experimentally to arrange that an equal intensity of ionisation in the meter is produced by these two beams, so that $q_{0}=q_{1}$.

Hence, for this particular case we may say

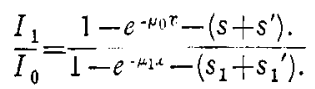

Now if the terms involving the abscrption ccefficients are more significant than those involving the scattering coefficients-and this can be arranged experimentally -we may write

$$
\frac{I_{1}}{l_{1}}=\frac{1-e^{-\mu_{0} r}}{1-e^{-\mu_{1} \lambda^{2}}}
$$


The above expression shows that if we wish to compare the intensities of two beams of different wave-lengths, then this can only be done if we know the coeffi. cients of absorption of the medium.

It may be noted here that even if measurements are restricted to beams of $\mathrm{X}$-rays of a single wave-length, the ordinary ionisation chambers can hardly be said to measure the intensity because the bulk of the radiation is transmitted through

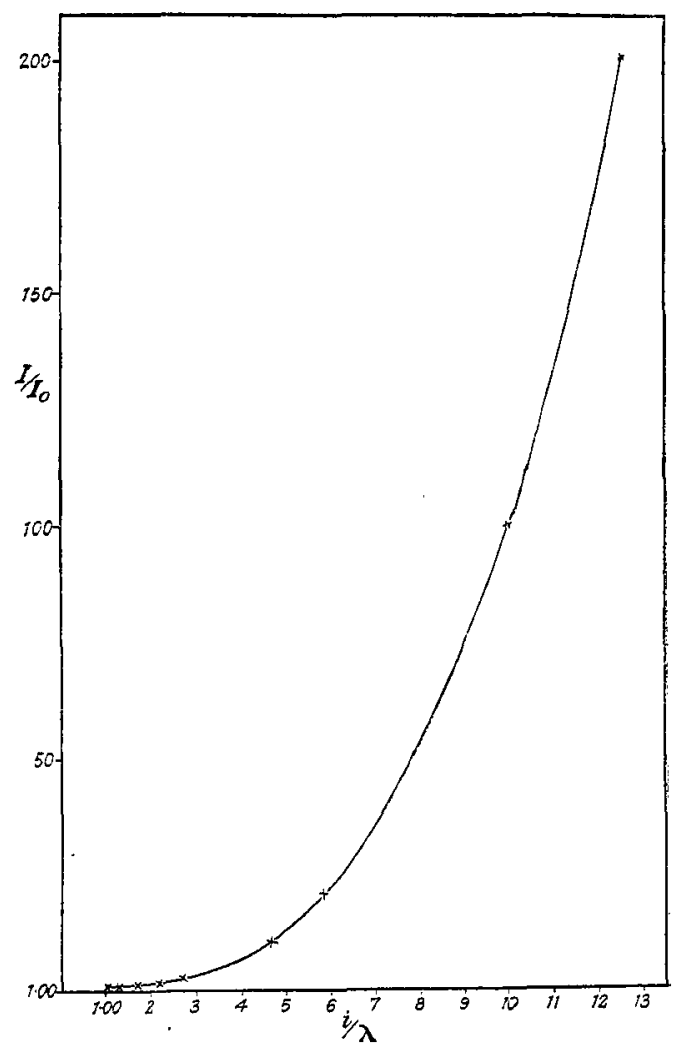

FIG. 2.

them, and so they should perhaps be more correctly looked upon as intensity-effect meters.

E. A. Owen has shown that a simple relation holds between the wave-length of a beam of X-rays and the absorption coefficient in a medium, provided that no "characteristic" radiation plays a part in the process of absorption. This relation is $\mu \propto \lambda^{5 / 2}$, and subsequent work has shown that $\mu \propto \lambda^{3}$ is of even more general application. By means of this last expression we can express the ratio $I_{1} / I_{0}$ in terms of the wave-lengths, and the graph of Fig. 2 shows how this ratio increases as the 
wave-length is shortened. The range of wave-length includes practically the whole extent of the radiation which is used in medical radiology.

The simple relationship outlined is only true under certain experimental conditions. They are, however, not too exacting to be practical. Perhaps the most important thing to bear in mind in designing an ionisation chamber suitable for comparisons of the intensity of different wave-lengths is that any secondary radiation produced in the instrument should not vitiate the results. There seems to be very little doubt that the simple air gap is the best for this purpose, for directly the rays are allowed to strike the solid walls of the chamber or the electrodes, secondary

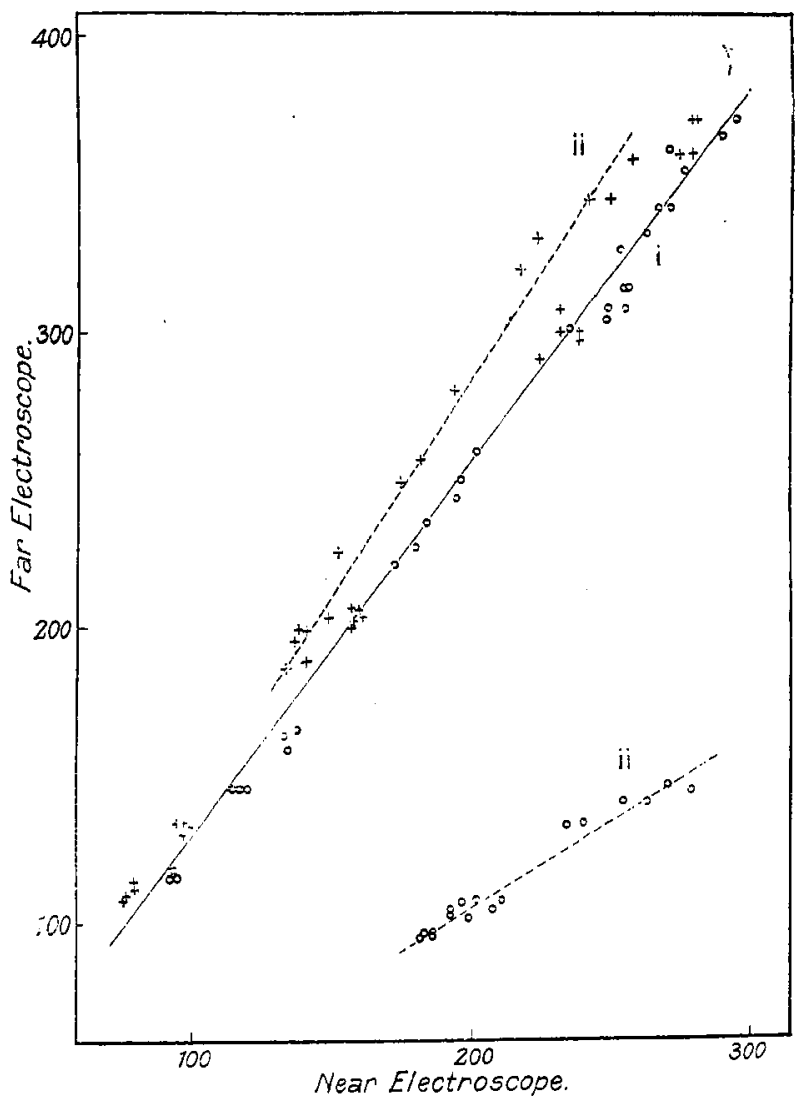

FIc. 3.

radiation may be produced by one wave-length but not by another, and so in this way true measurements are not obtained.

As an example of this, Fig. 3 shows two sets of experimental results, which were obtained in the following way:-

An $\mathrm{X}$-ray tube was set up in such a way that it could send a vertical beam to 
a near electroscope $(40 \mathrm{~cm}$.) and a horizontal one to a far electroscope (4 metres). In experiment No. 1 the near electroscope consisted of a simple air gap cut transversely in a lead cylinder, the electrodes being set so that the vertical beam of X-rays. just missed them. The ionisation in this gap was measured by means of a gold leaf connected electrically to one of the electrodes. The far electroscope consisted of an ordinary gold leaf electroscope made of lead fitted with a central brass stalk holding the leaf and a winclow of thin aluminium to allow the $\mathrm{X}$-rays to enter the instrument.

Simultaneous readings were obtained upon the two electroscopes with (I) a beam of "soft "X-rays and (2) a beam of " hard" rays.

It was easy to arrange that the near electroscope gave identical readings for the " hard " and "soft " rays-but when this was the case the reading of the far electroscope was considerably greater with "hard" than with "soft" rays until a diaphragm was used to cut down the radiation so that identical readings were also obtained on the far electroscope with " hard " and "soft " rays. This is shewn by the single full line I going through the crosses (" soft "X-rays) and circles ("hard" $\mathrm{X}$-rays) (Fig. 3). In experiment No. 2 the only change made was in the near electroscope. A paper cylinder $3.5 \mathrm{~cm}$. long and $1.5 \mathrm{~cm}$. diameter, fitted with a central electrode of aluminium, was substituted for the simple air-gap. Exactly the same thing was now carried out with this arrangement, and the two sets of experimental valucs show that the composition of this ionisation chamber has greatly favoured the soft type of radiation. It will be seen that in order to get an equality of readings with "hard " and "soft" X-rays on this ionisation vessel it was necessary to use a beam of " hard" rays about $2 \frac{1}{2}$ times as intense as would have been sufficient had the simple air-gap been used.

\section{CONCLUSTON.}

Little remains for me to say about the necessity which exists for a method of comparing and measuring the intensities of beams of X-rays of different wavclengths.

In the practice of medical radiology a variety of indicators is used. Each has its use within restricted limits, but no one of them can at the present time be looked upon with complete confidence to give the information desired.

In purely scientific work, each one puts up some method which serves the purpose in hand, but it is almost impossible at present for any research centre to. make use of the data given by other centres. For these reasons I hope that this combined meeting of the Physical and Röntgen Societies (February 23, 1923) may mark the beginning of an attempt to set up an unequivocal method of comparing. and measuring the intensities of beams of X-rays of different wave-lengths, and that it may then be possible to specify some quantity which shall be looked upon as an international unit of X-ray energy. 